\title{
Prolog: A Linguagem, A Máquina Abstrata de Warren e Implementações
}

\author{
George Souza Oliveira ${ }^{1}$ \\ Anderson Faustino da Silva ${ }^{1}$
}

\begin{abstract}
Resumo: A principal motivação para o uso de programação em lógica é permitir que os programadores descrevam o que eles querem separadamente de como alcançar este objetivo. Neste contexto PROLOG é a linguagem de programação em lógica mais popular, tendo a máquina abstrata de Warren como o seu modelo de execução padrão. A escolha desta máquina como modelo de execução ocorreu devido ao fato desta oferecer várias vantagens, tais como fácil compilação, portabilidade e código compacto. Este tutorial tem por objetivo descrever as características principais da linguagem PROLOG, do seu modelo de execução e apresentar os principais sistemas PROLOG.
\end{abstract}

\begin{abstract}
The main motivation for using logic programming is to allow programmers to describe what they want separately of how to achieve this goal. In this context PROLOG is the most popular logic programming language, and the Warren Abstract Machine is its default execution model. The choice of this machine as a standard occurred due to the fact that it offers several advantages, such as portability and compact code. This survey describes the main features of the PROLOG language, its execution model, and several PROLOG systems.
\end{abstract}

\section{Introdução}

Linguagens que implementam programação lógica [1, 2] possuem uma clara correspondência à lógica matemática e expressam a computação sem descrever o seu fluxo de controle. Em outras palavras, a descrição do programa é baseada em "o que" realizar, em vez de "como" realizar, ao contrário do que ocorre na programação imperativa [3]. Isso é baseado na premissa de que qualquer algoritmo consiste de uma especificação lógica (a descrição das propriedades do resultado esperado) e um controle (a forma de executar esta especificação). De posse da especificação lógica, o próprio sistema é encarregado de prover o controle e encontrar a solução do problema (caso esta exista). Esse estilo de programação torna as linguagens lógicas tanto mais fáceis quanto mais difíceis se comparadas a outras estratégias de

\footnotetext{
${ }^{1}$ Departamento de Informática

Universidade Estadual de Maringá

Avenida Colombo, 5790 - Bloco C56 - Maringá/PR - CEP 87020-900

\{geo.soliveira@gmail.com, anderson@din.uem.br\}
} 
programação. Fácil porque a forma de encontrar a solução fica a cargo da própria linguagem, e difícil porque esquemas de programação mais comuns não são suportados.

A primeira linguagem de programação lógica surgiu no início dos anos 70 [4] como um provador de teoremas especializado, que eventualmente evoluiu para uma linguagem mais poderosa chamada PROLOG [2]. Esta última descreve a programação como um subconjunto de lógica de primeira ordem, as cláusulas de Horn [5], sendo portanto, uma linguagem declarativa [6]. Esta característica torna PROLOG uma linguagem de programação simples e eficiente na implementação dos conceitos de unificação e busca.

A implementação de linguagens declarativas, de PROLOG em particular, é uma tarefa difícil, visto que as máquinas tradicionais são sequenciais, uma característica não existente neste tipo de linguagem (pelo menos não de forma explícita). Além disso, como o controle de execução fica a cargo da própria linguagem, o desenvolvedor se vê obrigado a considerá-lo e projetá-lo. Isso significa que as estratégias empregadas para criação de compiladores ou interpretadores para linguagens imperativas não são suficientes para a criação de compiladores ou interpretadores para linguagens declarativas e, portanto, lógicas.

Uma alternativa para implementação de Prolog surgiu com a Máquina Abstrata de Warren (WAM) [7, 8], um modelo que permite compilar código PROLOG para uma representação intermediária sequencial. Com esse modelo, um ambiente PROLOG pode ser implementado tanto por hardware quanto por software. A implementação por hardware, no entanto, é apenas um conceito teórico, visto que é uma implementação difícil devido a alta granularidade das instruções WAM. Além disso, o desenvolvimento do hardware ao longo dos anos e o aumento do número de instruções com baixa granularidade para as arquiteturas atuais mostrou que o uso de tal abordagem é inviável. Mesmo que um hardware especializado fosse criado, seria difícil, mesmo para os mais adeptos, adquirir uma máquina que só executasse PROLOG. Por fim, a dificuldade em obter bom desempenho em hardware tradicional somado ao custo de implementação de hardware exclusivo justificam que ambientes PROLOG tenham sido essencialmente projetados por software.

Por outro lado, a implementação por software acarreta um alto overhead proveniente da interpretação de código WAM para código de máquina equivalente. Além disso, a alta granularidade das instruções WAM impedem a aplicação de diversas otimizações de código [9]. Uma alternativa é implementar PROLOG para a geração de código nativo. Os primeiros sistemas a utilizarem esta última abordagem foram Aquarius [10] e Parma [11], que utilizavam análise global [12] para especializar a unificação e otimizar a seleção de cláusulas. Embora essas implementações tenham demonstrado que a execução de PROLOG é comparável à das linguagens imperativas em alguns casos, as mesmas foram abandonadas por serem muito difíceis de manter.

Dessa forma, os sistemas Prolog mais comuns são atualmente implementados como 
interpretadores [13, 14, 15, 16, 17, 18]. Como alternativa, alguns deles também oferecem execução de código nativo, como GNU-Prolog [15], SICStus Prolog [13], CIAO [16], BinProlog [19] e XSB Prolog [17]. Em contrapartida, YAPc [20] emprega outra abordagem diferente, gerar código em tempo de execução para as regiões executadas mais frequentemente. YAPc foi projetado para ser utilizado com o interpretador YAP [14], a fim de torná-lo um ambiente de modo misto de execução. Um sistema deste porte possui a vantagem de utilizar informações coletadas em tempo de execução para especializar o código nativo a ser gerado, sem a necessidade de implementar um analisador global.

Este tutorial tem por objetivo descrever a estrutura básica da linguagem de programação PROLOG, o modelo padrão de sua execução juntamente com as principais estratégias propostas para melhorar o seu desempenho e por fim sistemas PROLOG.

O restante deste tutorial está organizado como segue. A Seção 2 apresenta as características principais da linguagem de programação PROLOG. A Seção 3 descreve a arquitetura do modelo de execução PROLOG - a WAM. Além disto, esta seção apresenta as principais estratégias apresentadas na literatura cujo objetivo é melhorar o desempenho da WAM. A Seção 4 apresenta as implementações PROLOG citadas no decorrer deste tutorial. E por fim, a Seção 5 encerra este tutorial com as considerações finais.

\section{A Linguagem Prolog}

ProlOG (PROgramming in LOGic) [21, 2] é uma linguagem de programação em lógica baseada em um subconjunto de lógica de primeira ordem, as cláusulas de Horn [5]. Cláusulas de Horn têm um algoritmo de busca eficiente que permite uma performance competitiva. Pode-se dizer que um programa PROLOG é uma base de dados composta de regras e fatos. Como tal, a linguagem é naturalmente declarativa: o desenvolvedor informa ao sistema como especificar o problema e o sistema, por sua vez, usa um algoritmo de busca para responder questões. Embora seja uma linguagem declarativa algumas características não lógicas foram adicionadas à PROLOG, tais como: controle de fluxo, entrada/saída e metaprogramação. As principais características da linguagem PROLOG são:

- É uma linguagem orientada ao processamento de símbolos, os chamado termos.

- Implementa uma lógica como linguagem de programação.

- Apresenta uma semântica declarativa inerente a uma lógica.

- Permite a definição de programas reversíveis, isto é, programas que não fazem distinção entre os argumentos de entrada e os de saída.

- Permite a obtenção de respostas alternativas. 
- Suporta naturalmente código recursivo e iterativo para a descrição de processos e problemas, dispensando mecanismos tradicionais de controle, tais como comandos de repetição.

- Permite associar o processo de especificação ao processo de codificação de programas.

- Representa programas e dados através do mesmo formalismo.

A principal utilização da linguagem PROLOG reside no domínio da programação simbólica, sendo especialmente adequada à solução de problemas envolvendo relações entre objetos. Exemplos de sua aplicação incluem processamento de linguagem natural [22] e construção de sistemas dedutivos [23].

Em PASCAL [24] ou em C [25], um programa é uma sequência de instruções a serem executadas uma após a outra. Já um programa em lógica se assemelha mais a um banco de dados, onde há várias informações e relações registradas, por exemplo do tipo: 'o homem é inteligente' ou 'X é inteligente se $\mathrm{X}$ é homem'. Uma segunda diferença no paradigma de programação em lógica é o conceito de consulta e de resposta de um programa. Nas linguagens convencionais, um programa começa sempre a partir do mesmo ponto de entrada. Na programação em lógica, a chamada é feita através de uma pergunta, ou consulta. E o resultado do programa são as condições necessárias para que a afirmação seja verdadeira.

Basicamente, um programa PROLOG é formado por um conjunto de cláusulas, que podem ser constituídas por fatos ou regras. O programa append cujo objetivo é concatenar duas listas pode ser escrito da seguinte forma:

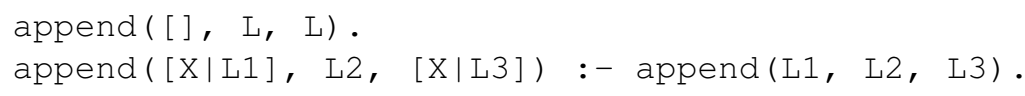

Este programa contém um fato e uma regra. Um fato consiste na declaração de uma cláusula que possui corpo vazio. Uma regra em PROLOG consiste em duas partes:

1. a cabeça, que é representada pelo predicado que se pretende provar, juntamente com seus argumentos. Na sintaxe de Edimburgo [26], o cabeça é separado do corpo pelo símbolo ":-".

2. o corpo, que consiste num conjunto de objetivos que devem ser satisfeitos a fim de que o predicado presente no cabeçalho seja verdadeiro.

Uma consulta em Prolog é sempre uma sequência de um ou mais objetivos. Para obter a resposta, o sistema Prolog tenta satisfazer todos os objetivos que compõem a 
consulta, interpretando-os portanto como uma conjunção. Satisfazer um objetivo significa demonstrar que esse objetivo é verdadeiro, assumindo que as relações que o implicam são verdadeiras no contexto do programa. Se a consulta contém variáveis, o sistema PROLOG deverá encontrar valores, que atribuídos às variáveis, satisfazem a todos os sub-objetivos propostos na consulta. A particular instanciação das variáveis com os valores que tornam o objetivo verdadeiro é a resposta. Se não for possível encontrar, no contexto do programa, nenhuma instanciação comum de suas variáveis que permita derivar todos os sub-objetivos propostos, o sistema informa que não há resposta. O sistema PROLOG aceita os fatos e regras como um conjunto de axiomas e a consulta do usuário como um teorema a ser provado. A tarefa do sistema é demonstrar que o teorema pode ser provado com base nos axiomas representados pelo conjunto das cláusulas que constituem o programa.

\subsection{Dados}

Os dados em Prolog e suas manipulações são modelados como lógica de primeira ordem. As variáveis lógicas representam tipos dinâmicos, as quais são atribuídos valores por meio de instanciação. Para identificar o objetivo mais comum entre dois objetos, PrOLOG utiliza unificação.

2.1.1 Variáveis Lógicas PROLOG é uma linguagem tipada dinamicamente: variáveis podem conter objetos de qualquer tipo em tempo de execução. Inicialmente o valor de uma variável é desconhecido: variáveis assumem valores através de instanciação. Variáveis podem ser passadas como argumentos para predicados ou como argumentos de dados compostos. Variáveis podem ser instanciadas apenas uma vez, contudo elas podem referenciar outras variáveis. Quando uma variável é instanciada, seu novo valor é visto por todas as variáveis que a referenciam.

2.1 Tipos Dinâmicos Tipos de dados compostos são novos tipos que podem ser criados em tempo de execução e variáveis podem conter valores de diferentes tipos. Tipos comuns são:

- átomos: constantes únicas, por exemplo: 1 , xyz;

- inteiros;

- listas: denotadas por colchetes, por exemplo [Cabeça | Cauda] ou $[1,2,3,4]$;

- estruturas: por exemplo: $f(X, L)$ ou $f(X, g(K))$.

Estruturas são similares às estruturas em $\mathrm{C}$ ou registros de $\mathrm{Pascal}$, elas possuem um nome (em PROLOG chamado de functor) e uma quantidade fixa de parâmetros (em PROLOG 
chamado de aridade).

2.1.3 Unificação Unificação [27] é uma operação de casamento de padrões que encontra a instância comum mais geral de dois objetos de dados. A operação de unificação é capaz de combinar componentes de objetos de qualquer tamanho em uma simples operação primitiva. Ligação de variáveis é feita através de unificação. Como uma parte do casamento de padrão, as variáveis no termo são instanciadas para torná-las iguais. Por exemplo, unificar $g(A, B, 1)$ e $g(K, 2, W)$ combina $A$ com $K, B$ com 2 e 1 com $W$.

\subsection{Controle}

Durante a execução, PROLOG tenta satisfazer as cláusulas na ordem em que elas estão listadas. Durante este processo, PROLOG utiliza uma execução para frente. Ele seleciona um literal, unifica e continua até não existirem mais literais. Quando o sistema invoca um predicado com mais de uma cláusula, PROLOG tenta satisfazer a primeira e constrói pontos de escolha para as outras. Se o sistema não pode tornar a cláusula verdadeira ocorre um retrocesso. O sistema retorna para o ponto de escolha mais recente e tenta a próxima cláusula. Neste processo, todas as ligações realizadas durante a tentativa de tornar a cláusula verdadeira são desfeitas, pois executar a próxima cláusula pode fornecer às variáveis diferentes valores. Note que em um dado caminho de execução a variável pode conter apenas um valor, mas em diferentes caminhos a variável pode conter valores diferentes. PROLOG é uma linguagem com uma única atribuição: se a unificação tentar fornecer à variável um valor diferente causará um falha e ocorrerá um retrocesso. Por exemplo: tentar unificar $g(1,2)$ e $g(A, A)$ causará uma falha porque os números 1 e 2 são diferentes.

PROLOG provê ainda mecanismos auxiliares para gerenciar o fluxo de controle, a saber:

- operação de corte,

- disjunção,

- condicional, e

- negação.

2.2.1 Operação de Corte A operação de corte é usada para evitar o retrocesso. Um corte no corpo de uma cláusula indica que esta foi a escolha correta, e que portanto o sistema não deve testar nenhuma outra cláusula para o mesmo predicado quando ocorrer retrocesso. Executar uma operação de corte tem o mesmo efeito em uma execução para frente de executar 
um true, ou seja, isto não possui nenhum efeito. Mas altera o comportamento do retrocesso. Por exemplo:

$$
\begin{aligned}
& g(A):-k(A), \quad !, f(A) . \\
& g(A)-p(A) .
\end{aligned}
$$

Durante a execução de $g(A)$, se $k$ (A) for verdadeiro então a operação de corte é executada. A operação de corte remove o ponto de escolha criado em $k$ (A) e o ponto de escolha que o sistema criou quando invocou $g(A)$. Como resultado, se $f(A)$ falha então o predicado $g(A)$ falha. Se não existisse a operação de corte e $f(A)$ falhasse ocorreria retrocesso primeiro para $\mathrm{k}(\mathrm{A})$, e se este também falhase ocorreria retrocesso para a segunda clásula do predicado $g$. Neste caso, apenas quando $p(A)$ falha o predicado $g(A)$ falha.

2.2.2 Disjunção A disjunção é uma maneira concisa de denotar uma escolha entre diferentes alternativas. Isto é mais conciso do que definir um predicado que tem cada alternativa como uma cláusula separada. Por exemplo:

$$
g(A):-(A=1 ; A=2 ; A=3) \text {. }
$$

Este predicado retorna três diferentes soluções e é equivalente a:

$$
\begin{aligned}
& g(1) \cdot \\
& g(2) . \\
& g(3) .
\end{aligned}
$$

A disjunção é freqüentemente usada em conjunto com a operação de corte.

2.2.3 Condicional Uma construção condicional, o if-then-else, é usada para denotar uma seleção entre duas alternativas em uma cláusula, quando é conhecida que se uma alternativa é tomada a outra não será necessária. Por exemplo: o predicado $g(A)$ pode ser escrito com uma construção if-then-else:

$$
g(A):-(k(A) \rightarrow f(A) ; p(A)) .
$$


Esta sintaxe tem semântica idêntica à definição anterior. A flecha $->$ em um if-thenelse atua como uma operação de corte que remove pontos de escolha até o ponto onde a operação if-then-else iniciou.

2.2.4 Negação PROLOG implementa negação como falha, denotado por $\backslash+$ ( ob jet i vo) . Isto não é realmente negação no sentido lógico, por isso o símbolo $\backslash+$ foi escolhido ao invés de not. Um objetivo negativo é verdadeiro se o objetivo falha, e falha se o objetivo é verdadeiro. Por exemplo:

$d(K):-\backslash+I(K)$.

O predicado $d(K)$ será verdadeiro se $I(K)$ falhar. Isto possui semântica idêntica a:

$d(K):-l(K)$, !, fail.

$d(K)$.

Se $l(K)$ é verdadeiro então fail causa uma falha, e o corte assegura que a segunda cláusula não será testada. Se $l$ (K) falha então a segunda cláusula é testada porque a operação de corte não é executada. Negação como falha nunca faz uma ligação de qualquer variável do objetivo que é negada. Isto é diferente da negação em lógica pura, que deve retornar todos resultados que não são iguais para aquele que satisfaz o objetivo. Negação como falha fornece resultados lógicos corretos se o objetivo negado não possui variáveis não instanciadas.

\subsection{Um Exemplo: Ordenação em Prolog}

Uma implementação do algoritmo de ordenação quicksort em PROLOG pode ser vista no código a seguir.

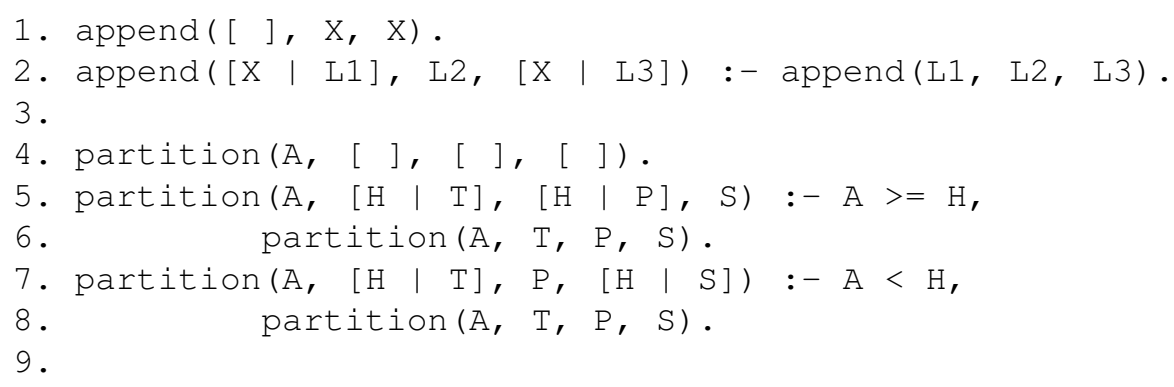




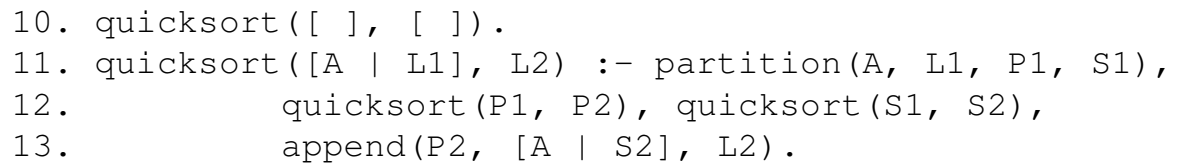

Este exemplo apresenta de forma clara como PROLOG descreve o programa e os dados utilizando o mesmo formalismo. De fato, um programa PROLOG descreve a estrutura de resposta esperada, ficando a cargo do sistema instanciar as devidas variáveis para que estas contenham uma resposta válida necessária, caso existam dados válidos na base de dados.

No programa quicksort apresentado nesta seção, a cláusula append apresenta como a resposta esperada pode ser formalizada pelo programador. $O$ fato localizado na linha 1 indica que a medida que o primeiro argumento seja instanciado com uma lista vazia, o segundo argumento deve ser retornado como resposta. A regra da linha 2 indica que o primeiro elemento da lista, que representa o primeiro argumento, estará contido no resultado. Além disto, esta regra também indica que o resultado é composto pela concatenação de duas listas, sendo que o primeiro elemento de uma das lista já foi inserido no resultado.

Uma característica interessante de PROLOG é o fato de não existir distinção entre argumentos de entrada e de saída. Desta forma, perguntas feitas à base de dados que contêm o programa quicksort pode ter as seguintes formas:

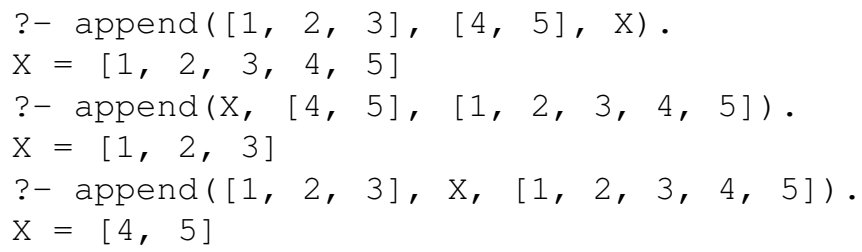

Esta característica descreve a diferença entre funções de linguagens imperativas e predicados da linguagem PROLOG. Funções possuem a clara noção entre argumentos de entrada e argumentos de saída, o que não ocorre na linguagem PROLOG.

Outra característica presente neste exemplo é o fato de PROLOG não possuir array. Desta forma, não existe uma maneira simples para acessar o elemento central e assim dividir uma lista em duas partes. Uma alternativa para resolver esta dificuldade é particionar os elementos a partir de um dos elementos da lista a ser ordenada. Assim, uma partição conterá além do elemento utilizado particionar a lista os elementos menores que este, enquanto a outra partição conterá os elementos maiores. Esta operação é realizada pelo predicado partition.

Portanto, o predicado quicksort primeiro particiona a lista a ser ordenada em duas partes, ordena cada sub-lista e então as concatena. 


\section{A Máquina Abstrata de Warren}

A Máquina Abstrata de Warren (WAM) é um modelo para execução de PROLOG criado por David Warren em 1983, que rapidamente se tornou padrão nas implementações desta linguagem. A motivação original deste projeto era implementá-lo em hardware [28], contudo esta idéia não obteve sucesso por duas principais razões: (1) o hardware deveria ser especializado para suportá-lo, limitando a sua procura no mercado, afinal há poucas pessoas interessadas em obter uma máquina que execute apenas PROLOG; e (2) por consequência de baixa procura, o hardware especializado teria pouco investimento frente aos demais microprocessadores.

Em contrapartida, implementações da WAM por meio de interpretadores [13, 14, 15, [16, 17, 18] se tornaram bastante populares devido à facilidade de implementação. Algumas destas implementações adquirem um bom desempenho, contudo, todas elas são prejudicadas pelo overhead intrínseco à tradução das instruções para a arquitetura em tempo de execução [29], além de limitarem a aplicação de otimizações devido a alta granularidade das instruções WAM. Implementações que geram código nativo alcançam um bom desempenho, mas tornam a transformação de código uma tarefa complexa, resultando em sistemas de difícil manutenção.

\subsection{O Estado Interno da WAM e a Organização da Memória}

Prolog é dinamicamente tipado, isto é, variáveis podem conter objetos de qualquer tipo em tempo de execução. Os termos da WAM são representados como palavras compostas por um rótulo que define o tipo do termo, e um valor que é utilizado para diferentes propósitos dependendo do seu tipo. Valores podem incluir inteiros, endereços de variáveis e termos compostos (listas ou estruturas). Variáveis indefinidas são implementadas como ponteiros para elas mesmas e são resolvidas durante a unificação, onde uma variável passa a apontar para outra. Essa forma de representação pode criar uma cadeia de ponteiros entre variáveis, sendo necessário percorrer toda a cadeia para encontrar o valor de uma variável qualquer. Essa operação é chamada de desreferenciação.

A WAM mantém o estado interno da computação atual definido por registradores. Os principais deles são:

- P: localização do programa (program counter).

- $\mathrm{CP}$ : apontador para a continuação de cláusulas (continuation pointer).

- E: apontador para o ambiente atual.

- B: apontador para o último ponto de escolha. 
- A: topo da pilha local.

- TR: topo da trilha.

- H: topo da pilha global (Heap).

- HB: valor de H no ponto de escolha mais recente.

- S: é usado pela unificação de termos compostos. Este registrador aponta para o argumento do termo que está sendo unificado: na WAM os argumentos são acessados através de sucessivos incrementos de $\mathrm{S}$.

- Mode: é usado para identificar o modo de execução de certas instruções, leitura ou escrita. Na WAM existem dois modos de execução: modo leitura e modo escrita. O modo de leitura ocorre quando o objeto unificado com a cabeça da cláusula é um termo já construído. O modo de escrita ocorre quando a cabeça da cláusula é unificada com um variável livre, e o termo precisa ser construído.

- $\mathrm{A}_{1}, \ldots, \mathrm{A}_{N}$ : registros dos argumentos.

- $\mathrm{X}_{1}, \ldots, \mathrm{X}_{N}$ : variáveis temporárias.

Os registradores $A_{i}$ e $X_{i}$ são, na verdade, idênticos; a diferença nos nomes meramente refletem usos diferentes. Os registradores $A_{i}$ são usados para passar os argumentos para um procedimento enquanto que os registradores $\mathrm{X}_{i}$ são usados para manter os valores de variáveis temporárias da cláusula.

Uma variável temporária é uma variável que tem sua primeira ocorrência na cabeça da cláusula e não ocorre em mais de um objetivo no corpo. Essas variáveis não precisam ser armazenadas no ambiente da cláusula. Por sua vez, uma variável permanente é qualquer variável não classificada como variável temporária. Variáveis permanentes são armazenadas em um ambiente e são endereçadas por deslocamentos do ponteiro de ambiente. Estas são referenciadas como $Y_{1}, Y_{2}$, etc. Variáveis permanentes são organizadas no seu ambiente de tal forma que elas podem ser descartadas na medida em que não são mais necessárias.

A respeito ao estado externo, a WAM dispõe de cinco regiões na memória que consistem de quatro pilhas para tratamentos específicos e uma área de código contendo instruções e dados relativos ao programa em si. As pilhas se expandem no momento da chamada de uma cláusula e se contraem no retrocesso, são elas: pilha global, pilha local, trilha e a PDL (push down list). A Figura 1 apresenta a organização da memória na WAM.

A pilha global (ou heap) armazena as estruturas compostas do programa PROLOG, isto é, listas e estruturas. A pilha local contém dois tipos de objetos: os ambientes e os pontos de escolha. Um ambiente normalmente está associado a uma cláusula e armazena todas as 


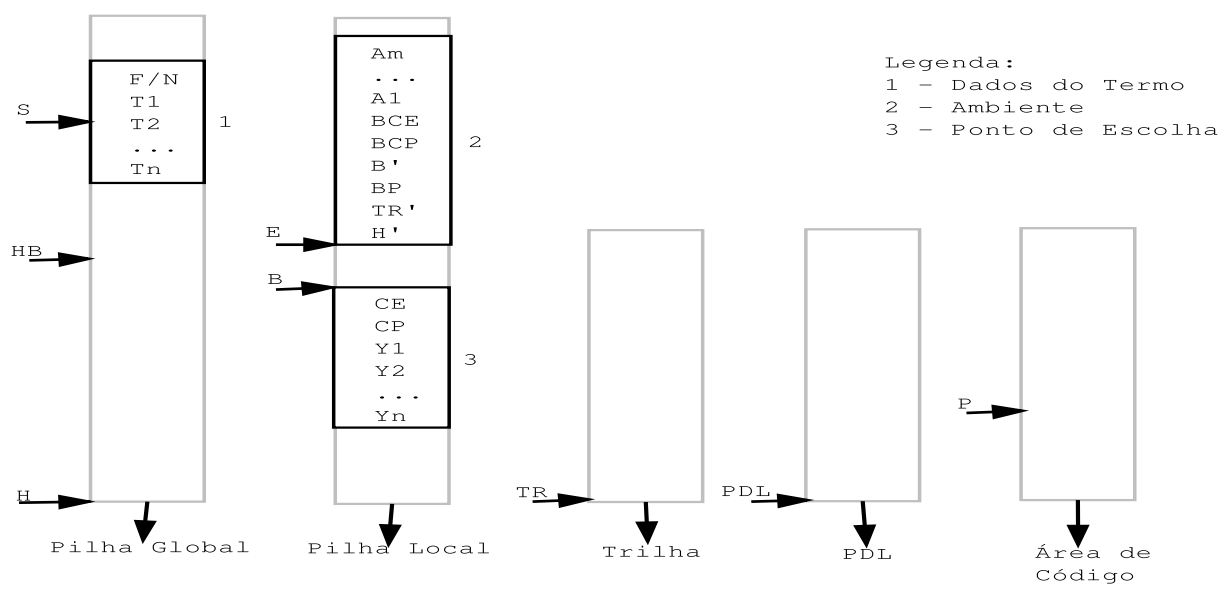

Figura 1. Organização da memória na WAM.

variáveis locais desta cláusula enquanto que os pontos de escolha guardam informações referentes ao estado de execução, a fim de possibilitar o retrocesso. A trilha armazena referências de variáveis que foram definidas durante a unificação, mas que devem ser indefinidas durante o retrocesso e a PDL auxilia na unificação dos termos.

\subsection{Conjunto de Instruções}

Os programas PROLOG são codificados em instruções sequenciais. Há, basicamente, uma instrução para cada símbolo do programa. Uma instrução consiste de um código de operação, ou simplesmente opcode, mais os operandos, que podem ser inteiros, deslocamentos ou endereços. A maioria das instruções PROLOG possui dois operandos. O modelo original da WAM consistia dos seguintes grupos de instruções: instruções get, instruções put, instruções unify, instruções de procedimento e instruções de indexação.

As instruções get são responsáveis por unificar os argumentos do predicado com os valores armazenados nos registradores A. As principais instruções deste grupo estão descritas a seguir. É necessário salientar que na descrição de todas as instruções, $A_{i}$ representa $o$ registrador de argumento $i, C$ e $F$ representam, respectivamente, uma constante e um functor e $\mathrm{V}_{n}$ representa tanto uma variável temporária $\left(\mathrm{X}_{n}\right)$ quanto uma variável permanente $\left(\mathrm{Y}_{n}\right)$.

get_variable $\mathbf{v}_{n}, \mathbf{A}_{i}$ insere o valor de $\mathrm{A}_{i}$ em $\mathrm{V}_{n}$;

get_value $\mathbf{V}_{n}, \mathbf{A}_{i}$ unifica o valor do registrador $\mathrm{A}_{i}$ com o conteúdo de $\mathrm{V}_{n}$, armazenando 
o resultado da unificação em $\mathrm{V}_{n}$, se $\mathrm{V}_{n}$ for uma variável temporária;

get_constant $\mathbf{C}, \mathbf{A}_{i}$ obtém o valor de $\mathrm{A}_{i}$ e desreferencia-o. Se o resultado for uma variável, esta variável recebe $\mathrm{C}$. Caso contrário, ocorrerá um retrocesso se este resultado for diferente de C;

get_nil $\mathbf{A}_{i}$ executa da mesma forma que get_constant $\mathrm{C}, \mathrm{A}$, mas neste caso a constante é [ ];

get_structure $\mathbf{F}, \mathbf{A}_{i}$ desreferencia o valor em $\mathrm{A}_{i}$ e prossegue conforme os casos a seguir: se o resultado for uma variável, esta recebe um novo ponteiro de estrutura que aponta no topo da heap, o functor $\mathrm{F}$ é inserido na heap e a execução prossegue em modo de escrita; se o resultado for um functor idêntico a $\mathrm{F}$, o registrador $\mathrm{S}$ é ajustado para apontar nos argumentos da estrutura e a execução prossegue em modo de leitura. Qualquer outro caso diferente resulta em retrocesso.

get_list $\mathbf{A}_{i}$ desreferencia o valor em $\mathrm{A}_{i}$ e prossegue conforme os casos a seguir: se o resultado for uma variável, esta recebe um novo ponteiro apontando para o topo da heap e a execução prossegue em modo de escrita; se o resultado é uma lista, o registrador S é ajustado para apontar nos argumentos da lista e a execução prossegue em modo de leitura. Qualquer outro caso diferente resulta em retrocesso.

A instrução get_variable $V_{n} A_{i}$ é utilizada se $V_{n}$ não estiver instanciada, ou seja, ela é utilizada somente na primeira ocorrência da variável na cláusula. Caso contrário, a instrução get_value $\mathrm{V}_{n}, \mathrm{~A}_{i}$ é utilizada.

As instruções put são responsáveis por armazenar os argumentos dos predicados nos registradores A. As instruções deste grupo são:

put_variable $\mathbf{Y}_{n}, \mathbf{A}_{i}$ cria uma referência para uma variável permanente não instanciada, representada por $\mathrm{Y}_{n}$, armazenando-a em $\mathrm{A}_{i}$ e $\mathrm{Y}_{n}$;

put_variable $\mathbf{x}_{n}, \mathbf{A}_{i}$ cria uma variável não instanciada na heap, armazenando-a em $\mathrm{A}_{i}$ e $\mathrm{X}_{n}$;

put_value $\mathbf{V}_{n}, \mathbf{A}_{i}$ insere o valor de $\mathrm{V}_{n}$ no registrador $\mathrm{A}_{i}$;

put_unsafe_value $\mathbf{Y}_{n}, \mathbf{A}_{i}$ insere o valor de $\mathrm{Y}_{n}$ no registrador $\mathrm{A}_{i}$ e globaliza $\mathrm{Y}_{n}$;

put_const $\mathbf{C}, \mathbf{A}_{i}$ insere a constante $\mathrm{C}$ no registrador $\mathrm{A}_{i}$;

put_nil $\mathbf{A}_{i}$ insere a constante [] no registrador $\mathrm{A}_{i}$; 
put_structure $\mathbf{F}, \mathbf{A}_{i}$ inicializa uma estrutura movendo o functor $F$ para uma nova posição na heap e atualiza $A_{i}$ com o ponteiro da posição criada. A execução pressegue em modo de escrita;

put_list $\mathbf{A}_{i}$ insere no registrador $\mathrm{A}_{i}$ o ponteiro da lista correspondente ao topo da heap e prossegue a execução em modo de escrita.

A instrução put_unsafe_value é utilizada no lugar de put_value para tratar variáveis inseguras que aparecem no último objetivo da cláusula. Uma variável insegura é uma variável permanente cuja primeira ocorrência não acontece em estruturas ou na cabeça da cláusula, isto é, é uma variável outrora inicializada pela instrução put_variable. A falta de um tratamento especial para tais variáveis permitem que estas mantenham referências para uma célula dentro de um ambiente mesmo após a sua exclusão pela instrução deallocate, produzindo uma cadeia de referências que podem levar a um valor indeterminado para um registrador A. Dessa forma, put_unsafe_value garante que a variável seja desreferenciada para algo externo ao ambiente atual, instanciando uma variável na heap para ela.

As instruções unify são precedidas por uma instrução get ou put que lêem ou escrevem estruturas ou listas. Isso significa que tais instruções computam conforme o modo de execução autal: modo de leitura ou modo de escrita. Basicamente, em modo de leitura, as instruções unify realizam a unificação de argumentos sucessivos de uma estrutura existente, endereçada pelo registrador $\mathrm{S}$ enquanto que em modo de escrita elas constroem os argumentos sucessivos de uma nova estrutura, endereçada pelo registrador H. As instruções são:

unify_void $\mathbf{N}$ salta $\mathrm{N}$ argumentos a partir de $\mathrm{S}$ quando está em modo de leitura. Em modo de escrita, esta instrução insere $\mathrm{N}$ novas variáveis (indefinidas) na heap;

unify_variable $\mathbf{V}_{n}$ armazena o argumento apontado por $\mathrm{S}$ em $\mathrm{V}_{n}$ quando está em modo de leitura. Em modo de escrita, a instrução insere uma nova variável (indefinida) na heap, e armazena sua referência em $\mathrm{V}_{n}$;

unify_value $\mathbf{v}_{n}$ unifica o argumento apontado por $\mathrm{S}$ com o valor contido em $\mathrm{V}_{n}$ quando está em modo de leitura. Além disso, se $\mathrm{V}_{n}$ for um temporário, o resultado da unificação é armazenado nela. Por outro lado, em modo de escrita, a instrução armazena o valor da variável $\mathrm{V}_{n}$ na heap;

unify_local_value $\mathbf{v}_{n}$ processa da mesma forma que unify_value $\mathrm{V}_{n}$, exceto que no modo de escrita, o valor de $\mathrm{V}_{n}$ é desreferenciado e é somente inserido na heap se o resultado não for uma referência para uma variável na pilha local. Caso o resultado for uma referência para uma variável na pilha local, uma nova variável (indefinida) é 
inserida na heap e a variável na pilha local é ajustada para apontar para esta nova variável. Caso $\mathrm{V}_{n}$ for uma variável temporária, $\mathrm{V}_{n}$ também é ajustado para apontar para esta nova variável.

unify_constant C operando em modo de leitura desreferencia o argumento apontado por S e, sendo esta uma variável, armazena nela a constante C. Se não for uma variável, o valor é comparado com $\mathrm{C}$ e, sendo diferente, o retrocesso ocorre. Em modo de escrita, a constante $\mathrm{C}$ é simplesmente inserida na heap.

A instrução unify_void $\mathrm{N}$ representa uma sequência de variáveis de ocorrência única, ou seja, nenhuma variável é necessária para processar esta instrução.

As instruções de procedimento realizam a transferência de controle e alocação de ambiente associado com o predicado a ser chamado. As instruções que compõem este grupo são:

allocate aloca um espaço para um novo ambiente na pilha local;

deallocate : restaura o estado mais recente e retira o último ambiente da pilha local.

call Pred, N : finaliza um objetivo do corpo da cláusula e faz o program counter $\mathrm{P}$ apontar para o predicado Pred com aridade N;

execute Pred : finaliza o último objetivo do corpo da cláusula e faz o program counter P apontar para o predicado Pred;

proceed : finaliza a execução de uma cláusula, fazendo o program counter $\mathrm{P}$ receber o valor de $\mathrm{CP}$. A diferença desta instrução com execute é que esta finaliza a execução de uma cláusula que foi chamada por outra pela instrução call.

Por fim, as instruções de indexação interligam cláusulas diferentes que compõem um predicado e pela filtragem de um subconjunto dessas cláusulas que poderiam corresponder a uma chamada de procedimento dado. A indexação é baseada em uma chave que é o functor principal do primeiro argumento do procedimento (armazenado em $A_{1}$ ). As instruções deste grupo estão representadas a seguir. Aqui, L, Lv, Lc, Ll e Ls são endereços de cláusulas (ou conjuntos de cláusulas), e Table é uma tabela hash de tamanho N.

try_me_else L cria um novo ponto de escolha armazenando na pilha local o endereço $\mathrm{L}$ da próxima cláusula, os valores dos registradores $\mathrm{E}, \mathrm{CP}, \mathrm{B}, \mathrm{TR}$ e $\mathrm{H}$ e os argumentos do predicado, que estão armazenados nos registradores $A_{1}$ a $A_{n}$. Após isso, HB e B são ajustados com o ponteiro atual da heap e com o topo da pilha local, respectivamente; 
retry_me_else I atualiza o ponto de escolha atual com o endereço da próxima cláusula contido em L;

trust_me_else fail descarta o ponto de escolha atual e recupera o estado mais recente de B e HB;

try L cria um novo ponto de escolha armazenando na pilha local o endereço da próxima instrução, os valores dos registradores $\mathrm{E}, \mathrm{CP}, \mathrm{B}, \mathrm{TR}$ e H e os argumentos do predicado, que estão armazenados nos registradores $\mathrm{A}_{1}$ a $\mathrm{A}_{n}$. Após isso, $\mathrm{HB}$ e $\mathrm{B}$ são ajustados com o ponteiro atual da heap e com o topo da pilha local. Por fim, o program counter $\mathrm{P}$ é ajustado com o endereço L da próxima cláusula;

retry $\mathrm{L}$ atualiza o ponto de escolha atual com o endereço da próxima instrução e ajusta $\mathrm{P}$ com o endereço L da próxima cláusula;

trust I descarta o ponto de escolha atual e recupera o estado mais recente de B e HB. Por fim, o emphprogram counter P é ajustado com o endereço L da próxima cláusula;

switch_on_term Lv, Lc, Ll, Ls desreferencia o valor contido em $A_{1}$, e ajusta $P$ com um dos endereços Lv, Lc, Ll ou Ls dependendo se o resultado da desreferenciação for uma variável, constante, lista não vazia ou estrutura, respectivamente.

switch_on_constant $\mathbf{N}$, Table utiliza a constante armazenada $A_{1}$ como chave para encontrar o endereço que será armazenado no program counter $\mathrm{P}$. Essa busca é feita na tabela hash $\mathrm{Table}$, cujo tamanho é $\mathrm{N}$. Ocorrerá retrocesso se a busca falhar;

switch_on_structure $\mathbf{N}$, Table processa da mesma forma que switch_on_constant, porém a chave utilizada é o functor principal armazenado em $A_{1}$.

\subsection{O Modelo de Execução WAM Para o Programa Append}

Como apresentado na seção anterior os programas PROLOG são codificados para instruções WAM, as quais são então executadas pelo sistema. Portanto, interpretadores ProLOG não executam o código fonte diretamente, mas sim a sua representação em código WAM. 
O código WAM correspondente ao código PROLOG do programa append, apresentado na Seção 2 é como descrito a seguir.

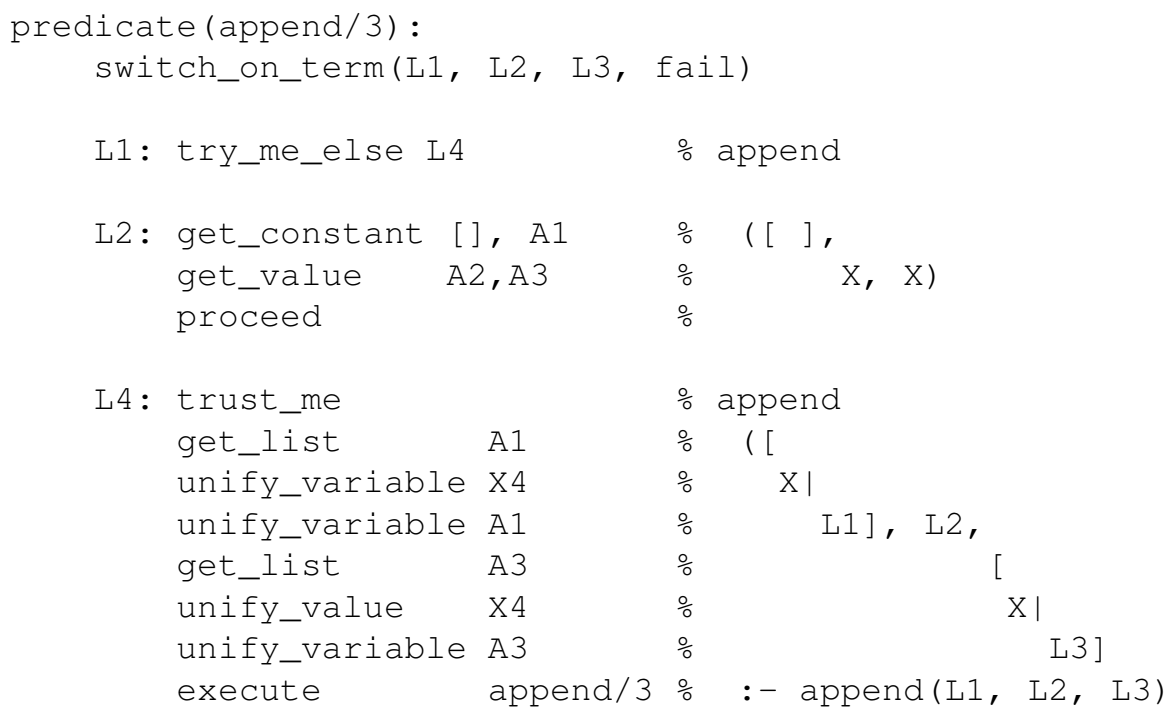

Como mencionado anteriormente, cada símbolo PROLOG foi transformado em uma instrução WAM. Observando este código WAM e a descrição de cada instrução é possível perceber que a WAM possui uma granularidade grossa. Desta forma, algumas instruções possui tarefas complexas, como por exemplo switch_on_term, que realiza uma operação de desreferenciação e atualiza o registrador $\mathrm{P}$ para apontar para a próxima instrução a ser executada.

\subsection{Melhorando o Desempenho da WAM}

A WAM foi um grande passo para a execução eficiente de PROLOG. A partir do ponto de vista de provas de teoremas, PROLOG é extremamente rápido. Mas existe ainda uma grande lacuna entre a eficiência de WAM e implementações de linguagens imperativas [30]. A seguir, são apresentadas algumas técnicas já propostas para melhorar ainda mais a execução de programas PROLOG em sistemas baseados na WAM.

3.4.1 Especializar Unificação O esquema de compilação de termos compostos na WAM foi projetado para ser completo e compacto, sem levar em conta questões de eficiência. $\mathrm{Na}$ verdade, as instruções de unificação padrão da WAM geram operações redundantes, principalmente porque a visita dos termos em estruturas compostas é feita em largura [31]. Além 
disso, o modo de escrita não é propagado para as subestruturas. De fato, sempre que uma estrutura é unificada em modo de escrita, é claro que todas as suas subestruturas também devem ser unificadas nesse modo. Não obstante, a WAM necessita utilizar várias variáveis temporárias no decorrer deste processo.

A alta granularidade das instruções WAM provém dessas características, visto que na unificação dos subtermos, as instruções devem testar o modo a cada momento. Isso evita a aplicação de otimizações para um determinado contexto do programa. O trabalho de Turk [32] descreve algumas otimizações para a WAM, inclusive um método para redução do overhead de manter o registrador de modo. Marien [33] apresenta um método para compilar unificação no modo de escrita que usa um número mínimo de operações. Uma linguagem intermediária para auxiliar nas operações de unificação da WAM foi introduzida por Van Roy [34], no custo de aumentar o tamanho do código. A eliminação das redundâncias nas operações de unificação só foram retiradas com o método proposto por Meier [31], onde os termos das estruturas eram visitados em profundidade. O método de Meier possibilitava ainda propagar o modo de escrita, além de utilizar menos variáveis temporárias que a WAM.

Dentre as implementações PROLOG atuais, YAP [14] implementa o método proposto por Meier. SICStus PROLOG [13, 35] emprega uma forma menos geral, onde somente os termos de listas são visitados em profundidade. Os demais sistemas não fornecem informações quanto a especialização das instruções de unificação.

3.4.2 Otimizar Seleção de Cláusulas Como apresentado na Seção 3.2. a WAM possui instruções que escolhem cláusulas dependendo da constante ou do functor principal do primeiro argumento. Porém, se todas as cláusulas dentro de um predicado contém functors (ou constantes) diferentes, então uma tabela hash precisa ser construída, a fim de possibilitar uma busca eficiente para a cláusula correta, além de evitar a criação de um ponto de escolha excedente (que seria inevitável sem esta estrutura). Isso significa que, no caso geral, os predicados podem ser compilados para criar no máximo um ponto de escolha entre o ponto de entrada e a execução da primeira cláusula [36, 37].

Esta característica facilita a implementação da seleção de cláusulas em sistemas baseados na WAM, mas muitos programas podem não se beneficiar desta estratégia, como por exemplo, em programas cuja seleção depende de mais de um argumento. Nesse sentido, um algoritmo ideal de seleção de cláusula deveria gerar código com as seguintes propriedades [30]:

- Ele testa somente as cláusulas que podem ser invocadas, baseado nos tipo dos argumentos;

- Ele evita todas as criações inúteis de pontos de escolha; 
- Seu tamanho é linear com o tamanho do programa;

- Ele cria pontos de escolha de forma incremental, isto é, os pontos de escolha contém somente partes do estado de execução que necessitam ser salvos;

- A degradação do desempenho é gradual conforme a insuficiência da informação sobre os tipos.

Infelizmente, não existe um método publicado que satisfaça todas essas condições. O que existe são algoritmos que satisfazem algumas delas e realizam melhor seleção de cláusulas que a WAM. Van Roy et al. [38] apresentaram um algoritmo que gera uma árvore de seleção ingênua e cria pontos de escolha de forma incremental. Uma forma mais restrita desta técnica, chamada shallow backtracking, foi implementada por Carlsson nas primeiras versões do sistema SICStus PROLOG [35]. Para isso, o conjunto de instruções foi extendido com uma instrução especial chamada neck. A versão atual de SICStus implementa uma versão ainda mais simples desta técnica [13].

Hickey e Mudambi [39] apresentaram um algoritmo para gerar uma árvore de testes de modo a minimizar o overhead do retrocesso. No pior caso, o tamanho da árvore tem uma proporção quadrática ao tamanho do código. Além disso, seu trabalho cria pontos de escolha de forma incremental, possibilitando que o coletor de lixo recupere mais memória. Um ano mais tarde, Kliger e Shapiro [40] utilizaram grafo acíclico direcionado para atingir o mesmo propósito e garantiram que, independente de qualquer programa, a seleção de cláusulas sempre seria melhor que a seleção de cláusulas original da WAM.

O sistema Aquarius [10, 28] produz um grafo de seleção para disjunções contendo testes para unificações, tipo e comparações aritméticas. Duas transformações são utilizadas para possibilitar os testes: (1) type enrichment, que adiciona informações de tipo para um predicado que carece dela e (2) factoring que permite o sistema tomar vantagem de testes em variáveis realizando a unificação de um termo para todos as ocorrências dele. Parma [11] é outro sistema que implementa uma estratégia semelhante.

Outras implementações atuais de PROLOG, tais como SWI-Prolog [41, 18], XSB [42, 17] realizam a seleção de cláusulas baseada em múltiplos argumentos, mas requerem um esforço adicional por parte do programador para possibilitar este processo. Este esforço não é necessário em ilProlog [43], que emprega uma heurística em tempo de compilação para realizar a mesma tarefa. Infelizmente, este tipo de seleção sofre o risco de indexar argumentos de saída, cujo efeito é aumentar o tempo de compilação de forma desnecessária. De fato, análise global poderia evitar este risco, prevendo os modos de execução possíveis em tempo de compilação. Contudo, nenhum destes sistemas implementam análise global. Uma forma sofisticada de contornar este problema foi apresentada por Costa et al., com a indexação por demanda [44] que permite a indexação de predicados estáticos e dinâmicos 
baseados somente nos argumentos de entrada, sem necessitar de análise global ou qualquer intervenção por parte do programador. Atualmente este método é o principal esquema de seleção de cláusulas do sistema YAP [14].

3.4.3 Gerar Código Nativo Uma base teórica para acelerar a execução de programas PROLOG é representar o código em instruções simples. Isso diminui a granularidade e dá oportunidades para a aplicação de diversas otimizações de código. Os primeiros experimentos publicados utilizando esta idéia foram realizados em 1986 por Komatsu et al [45] e Tamura [46], que demonstraram que a chave para o alto desempenho de PROLOG não está associada necessariamente a um hardware especializado.

Por volta de 1988, Taylor e Van Roy trabalharam nos sistemas Parma [11] e Aquarius [10, 28] que compilam código PROLOG para nativo, utilizando análise global para fornecer informações para as otimizações. Ao contrário de Komatsu et al. e Tamura, Taylor e Van Roy ignoraram o uso da WAM, pois estavam confiantes que a baixa granularidade do conjunto de instruções permitiria que todas as otimizações fossem expressadas. Parma compilava código PROLOG utilizando uma representação intermediária de três endereços e Aquarius se baseava na Berkeley Abstract Machine (BAM), que continha mais instruções que WAM (porém mais simples), inclusive algumas especializadas em tempo de execução. O resultado não foi diferente, apesar da dificuldade de manutenção destes sistemas, Aquarius e Parma superavam outras implementações existentes.

Essa forma de execução, no entanto, não ficou restrita somente a esses sistemas. Atualmente, uma boa parte das implementações PROLOG geram código nativo, tais como GNUProlog [15], SICStus Prolog [13, 35], CIAO [16], BinProlog [19] e XSB [42, 17].

Uma variação dessa estratégia é a geração de código nativo em tempo de execução, com o uso de compiladores just-in-time (JIT). O único compilador JIT para YAP existente é o YAPc, desenvolvido para o sistema YAP por Silva em 2007 [20]. Com este compilador, as cláusulas mais frequentemente executadas de um programa são especializadas e otimizadas em tempo de execução. Resultados mostraram que alguns programas obtiveram uma redução do tempo de execução entre 2 a 7 vezes, sob uma execução que somente interpreta [20]. YAPc foi utilizado com um prova de conceito para execução eficiente de PROLOG e não chegou a integrar a arquitetura do YAP.

3.4.4 Utilizar Análise Global Tradicionalmente, análise global para programas lógicos é usada para derivar informações, como de tipo e modo, com o objetivo de aumentar a velocidade de execução e/ou reduzir o tamanho do código. Os algoritmos de análise global estudados até o momento são todos instâncias de um método geral chamado de interpretação abstrata [47], cuja idéia geral é executar o programa sobre um domínio simples, isto é, um domínio conservativo, a fim de satisfazer um pequeno conjunto de restrições. No término 
da execução, os resultados encontrados fornecem uma aproximação correta de informações sobre o programa analisado. Le Charlier et al [48, 49] realizaram um estudo extensivo de algoritmos e domínios de interpretação abstrata e sua efetividade em derivar tipos. Getzinger [50] apresentou recentemente uma taxonomia extensa de domínios de análises e estudou seus efeitos sobre o tempo de execução e tamanho do código.

As primeiras evidências de que análise global seria considerada útil para implementações Prolog vieram nos trabalhos de Mellish em 1981 e 1985 [47, 51], mas as primeiras medidas de praticidade foram publicadas em 1988 por Warren et al. [12], ao avaliar dois sistemas de análise global: $\mathrm{MA}^{3}$ e Ms. O trabalho concluiu que ambos os analisadores são efetivos em derivar tipos e não aumentam o tempo de compilação de forma considerável. Van Roy e Taylor também obtiveram bons resultados nos sistemas Aquarius [10, 28] e Parma [11]. Atualmente, poucos sistemas não-obsoletos empregam análise global no código, a exemplo, o sistema CIAO [16].

3.4.5 Utilizar Memoização Memoização (do inglês memoization) é uma técnica para manter em cache a solução de predicados já processados. A adição desta técnica no mecanismo de resolução de PROLOG permite obter um novo modelo de execução que realiza ambos execução de baixo para cima quanto de cima para baixo. Para certos algoritmos, como algoritmos de programação dinâmica, esse novo modelo permite executar definições lógicas com uma baixa complexidade, se comparado ao mecanismo tradicional da WAM.

Uma implementação de memoização é a resolução OLDT (Ordered Linear Resolution of Definite Clauses with Tabulation) [52] e uma generalização dela é a resolução SLG [53], que lida também com negação e foi implementada primeiramente no sistema XSB [42, 17]. Essa implementação executa código PROLOG com menos de $10 \%$ de overhead relativo a WAM e é muito mais rápido que sistemas de banco de dados dedutivos [54]. Memoização também é empregada pelo sistema YAP [55] e está em fase de implementação no sistema GNU-Prolog [15].

\section{Implementações Prolog}

Geralmente as implementações da WAM por software são uma ordem de magnitude mais lenta do que linguagens imperativas. Por um lado, o uso de interpretadores possui o atrativo de tornar o sistema portável e interativo. Por outro lado, compiladores conseguem gerar código mais eficiente. As implementações citadas neste trabalho são descritas a seguir e uma boa parte delas suportam ambos os modos de execução.

Aquarius [10, 28] foi desenvolvido por Peter Van Roy, sendo o primeiro sistema a compilar código nativo sem um estágio intermediário baseado na WAM. Aquarius adotava 
o modelo de execução BAM (Berkeley Abstract Machine) que, ao contrário da WAM, empregava instruções mais simples, o que permitia aplicar extensas otimizações. Outras características deste compilador incluem: (1) exploração do determinismo, que permitia simplificar o retrocesso por saltos condicionais; (2) especialização de unificações, simplificando a unificações para simples atribuições, quando possível e (3) análise de fluxo de dados, que fornecia informações necessárias para a exploração do determinismo e especialização da unificação. Aquarius mostrou que a execução de programas PROLOG pode competir com programas gerados por um compilador otimizador $\mathrm{C}$ para uma classe de programas não-triviais [28]. Contudo, em vista das técnicas empregadas, somado ao cuidado sobre algumas instruções da BAM (que necessitavam ser especializadas em tempo de execução), Aquarius se tornou um sistema muito complexo para ser mantido. Em consequência, Aquarius não teve continuidade sendo abandonado em 1993.

BinProlog [19] desenvolvido por Tarau é um sistema Prolog capaz de interpretar código, gerar código $\mathrm{C} / \mathrm{C}++$ ou ainda gerar código executável. BinProlog provê um alto nível de abstração para o desenvolvimento de aplicações distribuídas e para Internet. BinProlog provê uma interface para as linguagens C [25], C++ [56] e JAVA [57], além de TCL/TK [58].

CIAO [16] é um ambiente de programação multiparadigma desenvolvido na Universidade Politécnica de Madri. Este sistema foi inicialmente projetado para transformar código PROLOG para C [25], deixando para o compilador desta linguagem gerar o código executável para a arquitetura subjacente. Nas versões atuais, CIAO fornece um ambiente completo de desenvolvimento incluindo um interpretador, um compilador, uma IDE, um analisador estático de código, além de um pré-processador potente capaz de gerar diversas otimizações, inclusive paralelização automática. O sistema oferece ainda uma linguagem de declaração, que possibilita a inserção estática de informações de tipo e modo, fornecidas pelo próprio usuário. Estas informações são utilizadas para realizar diversas otimizações de alto nível, incluindo especialização abstrata múltipla [59], avaliação parcial [60] e redução de concorrência [61]. Na ausência de tais informações, o próprio sistema tenta inferi-las, utilizando os métodos propostos em [62], [63] e [64].

GNU-Prolog [15] é um sistema Prolog desenvolvido por Diaz et al, que teve início em 1996 com o nome de Calypso e que foi posteriormente lançado como um produto GNU com o nome de GNU-Prolog. O sistema fornece dois modos de execução a saber: interpretado e nativo. Embora o compilador gere código nativo de alta qualidade, um trabalho publicado recentemente demonstrou que o interpretador não tem um bom desempenho frente a outros sistemas interpretados, como CIAO, SICStus e YAP [65]. GNU-Prolog está em desenvolvimento para prover suporte a um coletor de lixo 
e a memoização. Metas de longo prazo estipulam a criação de um compilador mais sofisticado, além da integração com LLVM [66].

Parma [11] é um compilador PROLOG experimental para arquitetura MIPS, desenvolvido na Universidade New South Wales em Sidney, Austrália. O componente mais importante no desempenho do Parma é a fase de análise global, que examina o programa como um todo para reunir informações que são utilizadas durante a compilação do programa. Informações sobre as características operacionais do programa, como cadeias de desreferenciamento, são utilizadas para remover muitas operações inerentes da linguagem, que sequer eram tratadas em outras implementações. Em Parma, as instruções são compiladas utilizando uma representação intermediária de três endereços, embora o modelo de memória seja ainda similar ao da WAM. Além disso, Parma emprega uma forma de armazenamento de forma a reduzir o custo do retrocesso. Infelizmente, assim como Aquarius, Parma foi abandonado devido a sua difícil manutenção.

SICStus Prolog [13, 35] foi desenvolvido pelo Swedish Institute of Computer Science (SICS). A implementação deste sistema foi baseada na especifiação da WAM, porém implementa alguns recursos adicionais como o corte, predicados aritméticos, testes de tipo, além de ter suporte para coleta de lixo, corrotinas e manipulação de interrupções. SICStus Prolog ainda implementa instruções de teste aritméticos sofisticados, baseado numa variação mais simples do método shallow backtracking [67]. Indexação de cláusulas e retrocesso são tratados dentro das instruções call e execute, ao contrário da especificação original da WAM, em que havia instruções específicas para essas tarefas. SICStus pode executar código PROLOG em três modos de execução distintos: (1) interpretado, onde as cláusulas são representadas como termos, a fim de acelerar a atualização na base de dados, além de dar suporte para depuração de código; (2) nativo, para execução rápida e (3) emulado, que simplifica a carga do sistema e o gerenciamento de memória.

SWI-Prolog [41, 18] desenvolvido por Wielemaker, foi projetado para ser um sistema simples e compacto. SWI-Prolog possui compatibilidade com outros sistemas como CIAO e GNU-Prolog. Com o objetivo de ser um ambiente de desenvolvimento e acadêmico, possui um framework de desenvolvimento completo, incluindo uma IDE, um analisador e uma interface para diferentes linguagens de programação, como C [25], C++ [56] e JAVA [57]. Uma característica interessante é o fato deste sistema possuir um módulo para servidores web multithreaded, capaz de gerar código HTML [68], autorização HTTP [69], além de gerenciar sessões.

XSB [42, 17] é um sistema PROLOG que foi desenvolvido por diversas instituições, com o intuito de prover uma abordagem alternativa para criar sistemas de banco de dados dedutivos. XSB emprega memoização para evitar avaliações não-finitas e redundância na resolução dos predicados, que são inerentes ao algoritmo de inferência presente nas 
implementações-padrão de Prolog. Isso é importante no contexto de sistema, visto que o reuso de informações já computadas é uma boa abordagem para sistemas que manipulam grande quantidade de dados. A execução de programas neste sistema pode ser feita da forma interpretada e nativa. A execução interpretada é lenta, mas predicados compilados são considerados estáticos por XSB no momento da compilação, o que pode ser indesejável ao manipular muitos dados. Como uma alternativa para compilação, XSB permite predicados dinâmicos, cujo código pode ser modificado durante a execução.

YAP Yet Another Prolog [14] é uma implementação de PROLOG desenvolvida na Universidade do Porto desde 1985. Possuindo apenas o modo de interpretação, este sistema suporta paralelismo e threads e foi o primeiro a conter estas características juntamente com a memoização. YAP compila cada cláusula dos programas PROLOG para uma representação baseada na WAM, chamada YAAM [70], cuja principal diferença para a WAM está no modo como é realizada a unificação de termos compostos. YAP provê ainda um sistema de indexação dinâmica, capaz de indexar múltiplos argumentos e termos compostos. Esta última característica, juntamente com memoização, permite que YAP seja utilizado como outra alternativa para criação de sistemas de banco de dados dedutivos, assim como XSB Prolog. Silva e Costa [20] projetaram um compilador JIT - o YAPc - para ser utilizado com o YAP, a fim de tornar este último um ambiente de modo misto de execução. A principal vantagem desta junção está no fato do novo sistema poder utilizar informações coletadas em tempo de execução, tal como os tipos das variáveis, com o objetivo de especializar o código nativo a ser gerado. De tal forma, isso minimiza a necessidade de implementar um analisador global no sistema, enquanto mantém a simplicidade do código. O princípio básico de YAPc é o mesmo dos compiladores JIT desenvolvidos para outros sistemas JAVA, como Jikes [71, 72], JUDO [73] e Sun Hotspot [74, 75]. Cada região de código (cláusula) é instrumentada com contadores e então interpretada. Cada contador é incrementado na medida em que as unidades associadas são invocadas. Durante este processo, um perfilador coleta informações sobre o programa em execução. Dessa forma, ao atingir um certo limite de invocação, a unidade é selecionada para compilação. Por fim, o compilador JIT é acionado a fim de gerar código especializado baseado nas informações anteriormente coletadas.

Uma comparação do desempenho de alguns destes sistemas foi descrita no trabalho de Martins e Silva [65]. 


\section{Considerações Finais}

A principal motivação para o uso de programação em lógica é permitir que os programadores descrevam o que eles querem separadamente de como alcançar este objetivo. Isto é baseado na premissa de que qualquer algoritmo consiste de duas partes: uma especificação lógica, a lógica, e uma descrição de como executar esta especificação, o controle. Programas lógicos são declarações descrevendo propriedades do resultado esperado, com o controle para entender o sistema. A maior parte deste controle pode ser automaticamente provida pelo sistema, o que o mantém claramente separado da lógica.

Prolog [21, 2] é uma linguagem que foi originalmente criada para resolver problemas em linguagem natural. A semântica de PROLOG ataca um balanceamento entre eficiente implementação e completude lógica [31, 8]. Isto atenta para descrever a programação como um subconjunto de lógica de primeira ordem, que não é apenas um provador simples de teorema, mas também uma linguagem de programação usual devido à simplicidade e à implementação eficiente dos conceitos de unificação e busca.

A primeira implementação de PROLOG foi um interpretador desenvolvido por Roussel e Colmerauer na década de 70 [76]. Em 1977, David Warren criou o primeiro compilador de PROLOG, o DEC-10 PROLOG [77], que gerava código assembly para o DEC-10. A investigação na implementação de PROLOG continuou com a Máquina Abstrata de David Warren (a WAM), uma linguagem intermediária para a compilação de PROLOG. A WAM oferecia várias vantagens, tais como fácil compilação, portabilidade e código compacto. Por essas razões, a WAM se revelou uma forma eficiente e elegante de permitir a execução de programas PROLOG numa máquina sequencial e desta forma se tornou rapidamente como modelo para implementações de PROLOG.

A motivação original no projeto de máquinas abstratas era a construção de hardware para suportar PROLOG eficientemente. Porém as instruções WAM realizam operações muito complexas, como a unificação, enquanto o desenvolvimento das novas arquiteturas seguiu uma direção oposta, possuir um pequeno conjunto de instruções simples. As dificuldades em obter bom desempenho em arquiteturas tradicionais e o custo de desenvolver hardware para suportar PROLOG justificam que a WAM tenha sido principalmente usada em implementações por software. Estas implementações tradicionalmente compilam o código PROLOG para o código de uma máquina abstrata que depois é interpretado, como realizado em: YAP [14], SICStus Prolog [13, 35], SWI-Prolog [41, 18], GNU-Prolog [15], BinProlog [19] e XSB [42, 17].

Após o advento dos interpretadores para Prolog, os sistemas Aquarius [10] e Parma [11] provaram que em alguns casos, as linguagens lógicas podem ter desempenho comparável ao das linguagens imperativas. O bom desempenho destes sistemas é devido basicamente a dois fatores, a saber: a geração de código nativo e o uso de análise global. Contudo tais 
sistemas foram descontinuados devido à alta complexidade de manutenção.

A geração de código nativo para PROLOG é um problema complexo. Usualmente o problema é solucionado organizando a computação num conjunto de fases especializadas, como ocorre no sistema SICStus Prolog que transforma o código PROLOG em código WAM, para depois transformar em uma nova linguagem intermediária [78].

Uma alternativa a esta abordagem é a geração de código C a partir de PROLOG [79]. A filosofia desta abordagem é deixar o trabalho de geração de código, como também da otimização, para o compilador C. Outra alternativa é a geração de código nativo em tempo de execução, como ocorre no sistema YAP com uso de compilação JIT [20].

Cada abordagem possui alguns problemas inerentes. Primeiro, a complexidade de alguns sistemas os tornam difíceis de serem mantidos. Consequentemente, sistemas como Aquarius e Parma foram abandonados, não existindo mais uma atualização de tais sistemas. Segundo, o uso de análise global não consegue obter uma aceleração maior que três. Além disto está técnica não funciona para qualquer tipo de programa. Terceiro, o overhead dos interpretadores, como por exemplo o YAP, é baixo, desta forma os sistemas que geram código nativo não conseguem um desempenho consideravelmente melhor.

Utilizando uma abordagem totalmente diferente, Mercury [80,1] mudou a linguagem. O objetivo foi melhorar o desempenho de programas lógicos. Contudo, surgiu um novo problema: Mercury não é PROLOG. Consequentemente, nem todos programas PrOLOG são executados por Mercury.

Nos últimos anos ocorreram melhorias significativas na compilação de PROLOG, resultando no desenvolvimento de sistemas eficientes, como SWI-Prolog e YAP. Tais melhorias foram decorrentes das técnicas propostas para melhorar o desempenho do modelo padrão de execução Prolog: a Máquina Abstrata de Warren. Neste contexto, este tutorial apresentou as características principais da linguagem PROLOG, o modelo de execução padrão, técnicas para melhorar o desempenho do modelo padrão e sistemas PROLOG.

\section{Referências}

[1] Fergus Henderson and Zoltan Somogyi. Compiling Mercury to High-Level C Code. In Proceedings of the International Conference on Compiler Construction, pages 197212, London, UK, 2002. Springer-Verlag.

[2] Leon Sterling and Ehud Shapiro. The Art of Prolog: Advanced Programming Techniques. MIT Press, Cambridge, MA, USA, 2 edition, 1994.

[3] R. W. Sebesta. Concepts of Programming Languages. Addison Wesley, San Francisco, CA, USA, 2009. 
[4] Robert A. Kowalski. The Early Years of Logic Programming. Communications of the ACM, 31:38-43, 1988.

[5] Alfred Horn. On Sentences Which are True of Direct Unions of Algebras. Journal of Symbolic Logic, 16(1):14-21, March 1951.

[6] J. W. Lloyd. Practical Advantages of Declarative Programming. In Proceedings of the Joint Conference on Declarative Programming, volume 94, pages 1-15, 1994.

[7] Hassan Aït-Kaci. Warren's Abstract Machine - A Tutorial Reconstruction. MIT Press, Cambridge, MA, 1991.

[8] David H. D. Warren. An Abstract Prolog Instruction Set. Technical Report 309, Artificial Intelligence Center, SRI International, Menlo Park, U.S.A, October 1983.

[9] Steven S. Muchnick. Advanced Compiler Design and Implementation. Morgan Kaufmann Publishers, San Francisco, CA, USA, 1997.

[10] P. Van Roy. Can Logic Programming Execute as Fast as Imperative Programming? $\mathrm{PhD}$ thesis, Berkeley, California, USA, December 1990.

[11] Andrew Taylor. Parma - Bridging the Performance GAP Between Imperative and Logic Programming. Journal of Logic Programming, 29(1-3):5-16, October 1996.

[12] Richard Warren, Manuel V. Hermenegildo, and Saumya K. Debray. On the Practicality of Global Flow Analysis of Logic Programs. In Proceedings of the International Conference and Symposium on Logic Programming, pages 684-699, Seattle, Washington, USA, 1988.

[13] Mats Carlsson and Per Mildner. SICSTUS Prolog - The First 25 Years. Theory and Practice of Logic Programming, 12(1-2):35-66, January 2012.

[14] Vr Santos Costa, Ricardo Rocha, and Luamas. The YAP Prolog System. Theory and Practice of Logic Programming, 12(1-2):5-34, January 2012.

[15] Daniel Diaz, Salvador Abreu, and Philippe Codognet. On the Implementation of GNU Prolog. Theory and Practice of Logic Programming, 12(1-2):253-282, January 2012.

[16] M. V. Hermenegildo, F. Bueno, M. Carro, P. Lípez-García, E. Mera, J. F. Morales, and G. Puebla. An Overview of CIAO and its Design Philosophy. Theory and Practice of Logic Programming, 12(1-2):219-252, January 2012.

[17] Terrance Swift and David S. Warren. XSB: Extending Prolog with Tabled Logic Programming. Theory and Practice of Logic Programming, 12(1-2):157-187, January 2012. 
[18] Jan Wielemaker, Tom Schrijvers, Markus Triska, and Torbjn Lager. SWI-Prolog. Theory and Practice of Logic Programming, 12(1-2):67-96, January 2012.

[19] P. Tarau. A Compiler and a Simplified Abstract Machine for the Execution of Binary Metaprograms. In Proceedings of the Logic Programming Conference, pages 119-128, London, UK, UK, September 1991. Springer-Verlag.

[20] A. F. da Silva and Vr Santos Costa. Design, Implementation, and Evaluation of a Dynamic Compilation Framework for the YAP System. In Proceedings of the International Conference on Logic Programming, pages 410-424, Porto, Portugal, 2007. SpringerVerlag.

[21] Marco A. Casanova, Fernando A. C. Giorno, and Antonio L. Furtado. Programação em Lógica e a Linguagem Prolog. Edgard Blucher LTDA, São Paulo, Brasil, 1 edition, 1987.

[22] Luis Quintano and Irene Rodrigues. Using a Logic Programming Framework to Control Database Query Dialogues in Natural Language. In Proceedings of the International Conference on Logic Programming, pages 406-420, Seattle, WA, 2006. SpringerVerlag.

[23] C. R. Ramakrishnan, I. V. Ramakrishnan, and David S. Warren. Deductive Spreadsheets Using Tabled Logic Programming. In Proceedings of the International Conference on Logic Programming, pages 391-405, Seattle, WA, 2006. Springer-Verlag.

[24] Israel de Campo Avillano. Pascal Estruturado. Ciência Moderna, Rio de Janeiro, Brasil, 2 edition, 2006.

[25] Luis Manoel D. Damas. Linguagem C. Livros Técnicos e Científicos, Rio de Janeiro, Brasil, 1 edition, 2007.

[26] D. H. D. Warren. Applied Logic - Its Use and Implementation as a Programming Tool. $\mathrm{PhD}$ thesis, University of Edinburgh, April 1977.

[27] J. W. Lloyd. Foundations of Logic Programming. Springer-Verlay, New York, USA, 1 edition, 1987.

[28] P. Van Roy and Alvin M. Despain. High-Performance Logic Programming with the Aquarius Prolog Compiler. IEEE Computer, 25(1):54-68, January 1992.

[29] Theodore H. Romer, Dennis Lee, Geoffrey M. Voelker, Alec Wolman, Wayne A. Wong, Jean-Loup Baer, Brian N. Bershad, and Henry M. Levy. The Structure and Performance of Interpreters. In Proceedings of the International Conference on Architectural Support for Programming Languages and Operating Systems, pages 150-159, Cambridge, Massachusetts, USA, 1996. ACM Press. 
[30] P. Van Roy. 1983-1993: The Wonder Years of Sequential Prolog Implementation. "Journal of Logic Programming , 29(1-3):5-16, December 1994.

[31] Micha Meier. Compilation of Compound Terms in Prolog. In Proceedings of the North American Conference on Logic Programming, pages 63-79, Austin, Texas, United States, 1990. MIT Press.

[32] Andrew K. Turk. Compiler Optimizations for the WAM. In Proceedings of the International Conference on Logic Programming, pages 657-662, London, UK, UK, July 1986. Springer-Verlag.

[33] A. Marien. An Optimal Intermediate Code for Structure Creation in a WAM-based Prolog Implementation. Technical Report T1988:01, Katholicke Universiteit Leuven, Heverlee, Belgium, May 1988.

[34] P. Van Roy. An Intermediate Language to Support Prolog's Unification. In Proceedings of North American Conference on Logic Programming, pages 1148-1164, Cleveland, Ohio, USA, October 1989. MIT Press.

[35] H. Nässén. Optimizing the SICStus Prolog Virtual Machine Instruction Set. Technical Report T2000:01, Intelligent Systems Laboratory, Uppsala University, Uppsala, Sweden, March 2001.

[36] Mats Carlsson. Freeze, Indexing, and Other Implementation Issues in the WAM. In Proceedings of the International Conference on Logic Programming, pages 40-58. MIT Press, May 1987.

[37] P. Van Roy. A Prolog Compiler for the PLM. Technical Report UCB/CSD 84/203, University of California, Berkeley, California, USA, November 1984.

[38] P. Van Roy, Bart Demoen, and Yves D. Willems. Improving the Execution Speed of Compiled Prolog with Modes, Clause Selection, and Determinism. In Proceedings of the Theory and Practice of Software Development, volume 250, pages 111-125. Springer Berlin Heidelberg, March 1987.

[39] Timothy Hickey and Shyam Mudambi. Global Compilation of Prolog. Journal of Logic Programming, 7(3):193-230, November 1989.

[40] Shmuel Kliger and Ehud Y. Shapiro. From Decision Trees to Decision Graphs. In Proceedings of the North American Conference on Logic Programming, pages 97-116. MIT Press, October 1990. 
[41] Jan Wielemaker. An Overview of the SWI-Prolog Programming Environment. In Proceedings of the International Workshop on Logic Programming Environments, pages 1-16, Heverlee, Belgium, December 2003. Katholieke Universiteit Leuven.

[42] Konstantinos F. Sagonas, Terrance Swift, and David Scott Warren. The XSB Programming System. In Proceedings of the Workshop on Programming with Logic Databases, pages 164-195, Vancouver, British Columbia, Canada, October 1993.

[43] Remko Tronçon, Gerda Janssens, Bart Demoen, and Henk Vandecasteele. Fast Frequent Querying with Lazy Control Flow Compilation. Theory and Practice of Logic Programming, 7(4):481-498, July 2007.

[44] Vr Santos Costa, Konstantinos Sagonas, and Ricardo Lopes. Demand-driven Indexing of Prolog Clauses. In Proceedings of the International Conference on Logic Programming, pages 395-409, Porto, Portugal, 2007. Springer-Verlag.

[45] Hideaki Komatsu, Naoyuki Tamura, Yasuo Asakawa, and Toshiaki Kurokawa. An Optimizing Prolog Compiler. In Proceedings of the International Conference on Logic Programming, pages 104-115, Tokyo, Japan, 1987. Springer-Verlag.

[46] Naoyuki Tamura. Knowledge-based Optimization in Prolog Compiler. In Proceedings of ACM Fall Joint Computer Conference, pages 237-240, Dallas, Texas, United States, November 1986. IEEE Computer Society Press.

[47] Chris S. Mellish. The Automatic Generation of Mode Declarations for Prolog Programs. Logic programming for Intelligent Systems, 13(2-3):103-179, August 1981.

[48] B. Le Charlier, Olivier Degimbe, Laurent Michel, and P. Van Hentenryck. Optimization Techniques for General Purpose Fixpoint Algorithms - Practical Efficiency for the Abstract Interpretation of Prolog. In Proceedings of the International Workshop on Static Analysis, pages 15-26, London, UK, UK, September 1993. Springer-Verlag.

[49] B. Le Charlier, Kaninda Musumbu, and P. Van Hentenryck. A Generic Abstract Interpretation Algorithm and its Complexity Analysis. Technical report, Brown University, Providence, RI, USA, 1990.

[50] Thomas Walter Getzinger. Abstract Interpretation for the Compile-Time Analysis of Logic Programs. PhD thesis, Los Angeles, California, USA, September 1993.

[51] Chris S. Mellish. Some Global Optimizations for a Prolog Compiler. Journal of Logic Programming, 1:43-66, 1985.

[52] Terrance Swift and David Scott Warren. Compiling OLDT Evaluation: Background and Overview. Technical Report 92/04, SUNY Stony Brook, March 1992. 
[53] Weidong Chen and David S. Warren. Query Evaluation under the Well-founded Semantics. In Proceedings of the ACM SIGACT-SIGMOD-SIGART Symposium on Principles of Database Systems, pages 168-179, Washington, D.C., United States, 1993. ACM Press.

[54] Terrance Swift and David S. Warren. Performance of Sequential SLG Evaluation. In Proceedings of the Symposium on Logic Programming, pages 219-238. MIT Press, November 1993.

[55] Ricardo Rocha, Fernando M. A. Silva, and Vr Santos Costa. On a Tabling Engine That Can Exploit Or-Parallelism. In Proceedings of the International Conference on Logic Programming, pages 43-58, London, UK, UK, 2001. Springer-Verlag.

[56] Bjarne Stroustrup. Princípios e Práticas de Programação C++. Bookman, Porto Alegre, Brasil, 1 edition, 2011.

[57] Harvey Deitel and Paul Deitel. Java - Como Programar. Prentice Hall, Rio de Janeiro, Brasil, 8 edition, 2010.

[58] Clif Flynt. Tcl/Tk: A Developer's Guide. Elsevier Science, New York, USA, 1 edition, 2012.

[59] Germán Puebla and Manuel Hermenegildo. Implementation of Multiple Specialization in Logic Programs. In Proceedings of the ACM SIGPLAN Symposium on Partial Evaluation and Semantics-based Program Manipulation, pages 77-87, La Jolla, California, United States, 1995. ACM.

[60] Germán Puebla, Elvira Albert, and Manuel Hermenegildo. Abstract Interpretation with Specialized Definitions. In Proceedings of the International Conference on Static Analysis, pages 107-126, Berlin, Heidelberg, 2006. Springer-Verlag.

[61] Germuebla, Maria J. Garce la Banda, Kim Marriott, and Peter J. Stuckey. Optimization of Logic Programs with Dynamic Scheduling. In Proceedings of the International Conference on Logic Programming, pages 93-107, Cambridge, June 1997. MIT Press.

[62] K. Muthukumar and M. Hermenegildo. Compile-time Derivation of Variable Dependency Using Abstract Interpretation. Journal of Logic Programming, 13(2-3):315-347, July 1992.

[63] Huseyin Saglam and John P. Gallagher. Approximating Constraint Logic Programs Using Polymorphic Types and Regular Descriptions. Technical Report CSTR-95-17, Department of Computer Science, University of Bristol, Bristol, UK, UK, 1995. 
[64] Claudio Vaucheret and Francisco Bueno. More Precise Yet Efficient Type Inference for Logic Programs. In Proceedings of the International Symposium on Static Analysis, pages 102-116, London, UK, UK, 2002. Springer-Verlag.

[65] Alexandre Locci Martins and A. F. da Silva. Benchmarking Prolog Interpreters. IEEE Latin America Transactions, 9(7):1079-1086, December 2011.

[66] Chris Lattner and Vikram Adve. LLVM: A Compilation Framework for Lifelong Program Analysis \& Transformation. In Proceedings of the International Symposium on Code Generation and Optimization, pages 75-86, Palo Alto, California, 2004. IEEE Computer Society.

[67] Mats Carlsson. On the Efficiency of Optimising Shallow Backtracking in Compiled Prolog. In Proceedings of the International Conference on Logic Programming, pages 3-16, Lisbon, Portugal, June 1989. MIT Press.

[68] Samy Mauricio Silva. HTML 5 - A Linguagem de Marcação que Revolucionou. NOVATEC, Rio de Janeiro, Brasil, 1 edition, 2011.

[69] Chris Shiflett. HTTP Developer's Handbook. Smas Publishing, New York, USA, 1 edition, 2003.

[70] Ricardo N. S. LOPES. Execução de Prolog com Alto Desempenho. Master's thesis, Universidade do Porto, Porto, Portugal, Master thesis, Universidade do Porto, Portugal, Jul. 1996.

[71] M. Arnold, S. Fink, D. Grove, M. Hind, and P. F. Sweeney. Adaptive Optimization in the Jalapeño JVM. In Proceedings of the ACM Conference on Object-Oriented Programming, Systems, Languages, and Applications, pages 47-65, Minneapolis, Minnesota, United States, 2000. ACM.

[72] M. G. Burke, J.-D. Choi, S. Fink, D. Grove, M. Hind, V. Sarkar, M. J. Serrano, V. C. Sreedhar, H. Srinivasan, and J. Whaley. The Jalapeño Dynamic Optimizing Compiler for Java. In Proceedings of the ACM Conference on Java Grande, pages 129-141, San Francisco, California, United States, 1999. ACM.

[73] M. Cierniak, G.-Y. Lueh, and J. M. Stichnoth. Practicing JUDO: Java Under Dynamic Optimizations. In Proceedings of the ACM Conference on Programming language Design and Implementation, pages 13-26, Vancouver, British Columbia, Canada, 2000. ACM.

[74] T. Kotzmann, C. Wimmer, H. Mössenböck, T. Rodriguez, K. Russell, and D. Cox. Design of the Java HotSpot Client Compiler for Java 6. ACM Transactions on Architecture and Code Optimization, 5:1-32, 2008. 
[75] M. Paleczny, C. Vick, and C. Click. The Java Hotspot Server Compiler. In Proceedings of the Java Virtual Machine Research and Technology Symposium, pages 1-12, Monterey, CA, USA, 2001.

[76] A. Colmerauer, H. Kanoui, R. Pasero, and P. Roussel. Un Systeme de Communication Homme-machine en Fracais. Technical Report 72-18, Groupe Intelligence Artificille, Universite Aix-Marseille II, October 1973.

[77] David H. D. Warren. Implementing Prolog - Compiling Predicate Logic Programs. Technical Report 39-40, Department of Artificial Intelligence, University of Edinburgh, October 1977.

[78] R. C. Haygood. Native Code Compilation in SICStus Prolog. MIT Press, New York, USA, 1 edition, 1994.

[79] J. Morales, M. Carro, and M. Hermenegildo. Improved Compilation of Prolog to C Using Moded Types and Determinism Information. In Proceedings of the Colloquium on Implementation of Constraint and Logic Programming Systems, pages 197-212, 2003.

[80] Thomas Conway, Fergus Henderson, and Zoltan Somogyi. Code Generation for Mercury. In Proceedings of International Logic Programming Symposium, pages 242-256, Portland, Oregon, USA, December 1995. 